\title{
ÉTUDE DU LACHER INSTANTANÉ D'UNE RETENUE D'EAU DANS UN CANAL PAR LA MÉTHODE GRAPHIQUE
}

\section{R. RÉ}

Ingénieur aux Ateliers Neyret-Beylier \& Piccard-Pictet

Après l'exposé de la théorie du calcul graphique des régimes variables dans les canaux, qui a paru dans le numéro 1 et le numéro 2 de cette Revue, il a paru intéressant de donner à l'hydraulicien, amené à résoudre certains problèmes d'écoulement, un exemple sur lequel il puisse se rendre compte des modalités de mise en œuvre et des difficultés d'application de la méthode.

On sait, connaissant les conditions d'alimentation et d'évacuation d'un canal, déterminer les lignes de remous qui s'y établissent, én régime permanent. Que ces conditions viennent à varier et l'on se trouve en présence d'un problème à régime variable beaucoup plus difficile à résoudre. La nouvelle méthode graphique se propose précisément de poursuivre dans ce dernier cas l'évolution du phénomène tout au long du canal.

L'étude suivante, choisie pour illustrer l'exposé général de la méthode, se situe à l'origine de la vaste étude sur la traversée du Rhin par les Armées alliées qu'ont faite les Laboratoires d'hydraulique des Ateliers NEYRETBeylier et Piccard-Pictet; l'objectif de cette recherche était de prévoir l'ampleur des intumescences qu'entraînerait la rupiure, par l'ennemi, de un ou plüsieurs barrages sur le fleuve; à la veille du franchissement de ce dernier obstacle, l'Etat-Major allié désirait connaître, aussi précisément que possible, la propagation des ondes de crues au cas où l'ennemi utiliserait ce mode de défense. Avant de s'adresser au modèle réduit, pour répondre aux multiples questions du Commandement militaire, on a obtenu une première analyse du phénomène et une première orientation des recherches par une étude théorique et notamment une application de la máthode graphique. Le cas que nous allons examiner constitue une forme schématique de ce problème.

Un canal d'une largeur invariable de 250 mètres, d'unc pente uniforme de 0,9 minn. par mière, est obturé par un barrage qui crée, à son amont immédiat, une hauteur d'eau de $10 \mathrm{~m} .80$ (fig. 1). Avec une approximation largement suffisante pour le problème étudié, nn peut assimiler la surface de la retenue à un plan horizon- tal. Le barrage laisse échapper un débit constant qui s'organise à l'aval suivant le régime uniforme :

$$
\begin{aligned}
& \text { hauteur d'eau } h=1,80 \mathrm{~m} \text {. } \\
& \text { vitesse moyenne } U=1,60 \mathrm{~m} . / \mathrm{sec} \text {. }
\end{aligned}
$$

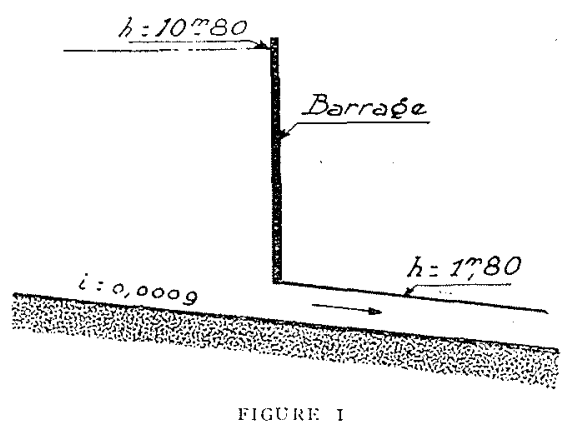

ce qui correspond à un coefficient de Chezy d'environ 40 et à un coefficient $\lambda=0,006$ en prenant pour $\lambda$ la définition $j=\frac{i \mathrm{U}^{2}}{g} \overline{\mathrm{R}}, \quad \lambda$ coefficient de perte de charge, $;$ perte de charge unitaire $=0,0009, \quad \mathrm{R}=$ rayon hydraulique. En raison de la grande largeur du canal, on peut confondre $\mathrm{R}$ et $h$.

On efface brusquement le barrage; quelles intumescences va-t-on enregistrer de part et d'autre du barrage, à des instanls donnés ?

Indépendamment de ces résultats, on s'étendra sur quelques détails graphiques de nature à faciliter la tâche des utilisateurs.

\section{RAPPEL DU PRINCIPE DE LA MÉTHODE}

$$
\begin{aligned}
& \text { I. - LOIS CARACTERISTIQUES } \\
& \text { DES OBSERVATEURS }
\end{aligned}
$$

a) Ondes élÉmentaires dans un canat. HoRIZONTAI. ET SANS PERTE DE CHARCE. - La base de la méthode, analogue à celle de Schnyder-Bergeron pour 
le calcul des coups de bélier, réside dans l'invariance (pour un canal horizontal et sans frottement) de la quantité $(U \pm 2 \mathrm{~V})$ pour des observateurs se déplaçant avec les ondes $(\mathrm{U}$ : vitesse moyenne, $\mathrm{V}=\sqrt{\mathrm{g} h}$; $h$ : hauteur d'eau, $g$ : accélération de la pesanteur; le signe + si l'observateur se trouve sur une onde descendante, le signe - si l'observateur se trouve sur une onde remontant le courant).

Dans un plan représentatif $(\mathrm{U}, \mathrm{V})$ les lois caractéristiques de ces observateurs sont des dioites de pente $\pm \frac{1}{2}$.

b) Ondes Élémentaires dans un CANal en PENTE AVEC PERTE DE CHARGE. - Si le canal a une pente $i$ et oppose à l'écoulement une résistance caractérisée par $\lambda$ l'observateur trouve alors que $U \pm 2 \mathrm{~V}$ n'est plus constant, mais varie cependant d'une façon connue.

Pendant un temps $d t$ il enregistre :

$$
d(\mathrm{U} \pm 2 \mathrm{~V})=\mathrm{g}\left(i-\lambda \frac{\mathrm{U}^{2}}{\mathrm{~V}^{2}}\right) d t
$$

Graphiquement, les observations s'enregistrent sur deux plans : le plan $x t$ et le plan $\mathrm{U}, \mathrm{V}$.

Soient deux observateurs $\mathrm{A}$ et $\mathrm{B}$ dont on connaît :

- pour A l'instant $t_{A}$ la position $x_{A}$; en ce lieu la vitesse est $U_{A}$ et la hauteur correspond à $V_{A}$.

- pour B : $t_{\mathrm{B}}-x_{\mathrm{B}}-\mathrm{U}_{\mathrm{B}}-\mathrm{V}_{\mathrm{B}}$

A descend le courant à la vitesse $U_{A}+V_{A}$.

$B$ remonte le courant à la vitesse $\left.\left|U_{B}-V_{B}\right|{ }^{1}\right)$.

Dans le plan $x t$, on trouve aisément la position $x_{C}$ et le temps $t_{C}$ où ils se rencontrent (fig. 2). Il suffit

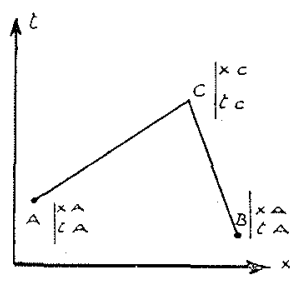

HGURE 2

de donner aux droites $\mathrm{AC}$ et $\mathrm{BC}$ des pentes correspondant aux vitesses de déplacement des deux observateurs respectifs.

Dans le plan U, V (fig. 3) le régime observé n'est plus situé sur les droites de pente $\pm \frac{1}{3}$ issues des points $A$ et $B\left({ }^{2}\right)$, mais sur ces droites décalées chacune des quantités $(U \pm 2 \mathrm{~V})$. Ainsi pour $\mathrm{A}$, on

(1) $\left|U_{13}-V_{B}\right|=$ en valeur absolue.

(2) Que nous désignerons encore un pein improprement sous le nom de droites caractéristiques.

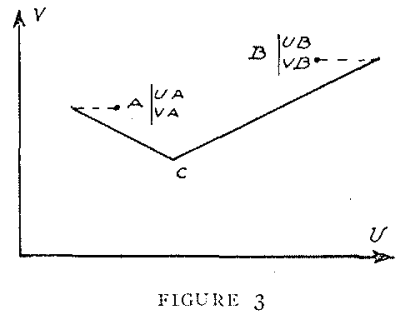

fera subir à sa caractéristique une translation parallèle

à $\mathrm{OU}$ et de valeur: $g\left(i-i \frac{\mathrm{U}_{\mathrm{A}}{ }^{2}}{\mathrm{~V}_{\mathrm{A}}{ }^{2}}\right)\left(t_{\mathrm{C}}-t_{\mathrm{A}}\right)$

$$
\text { pour } \mathrm{B}: \quad g\left(i-i, \frac{\mathrm{U}_{\mathrm{B}}{ }^{2}}{V_{\mathrm{B}}{ }^{2}}\right)\left(t_{\mathrm{C}}-t_{\mathrm{B}}\right)
$$

c) Ondes DE CHOC. - Les lois précédentes s'appliquent aux ondes élémentaires dont la combinaison constitue des mouvements progressifs des niveaux d'eau. Les ondes raides de grande amplitude ou ondes de choc obéissent à des règles différentes.

a) Ondes de choc descendant le courant. - C'est l'onde à front raide qui marque, pour toutes les sections aval, le début de la crue.

Cette onde possède une célérité

$$
\mathrm{W}=\mathrm{U}_{1}+\frac{\mathrm{V}_{2}}{\mathrm{~V}_{1}} \sqrt{\frac{\mathrm{V}_{1}{ }^{2}+\mathrm{V}_{2}{ }^{2}}{2}}
$$

$\mathrm{U}_{1} \mathrm{~V}_{1}$ étant relatifs au régime uniforme avant le passage de l'onde;

$U_{2} V_{2}$ constituant l'état laissé par l'onde (fig. 4).

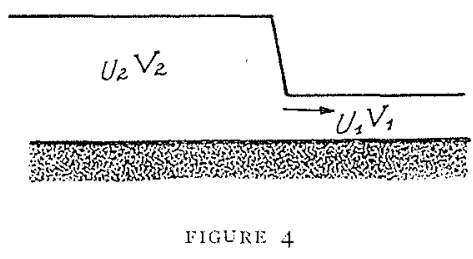

Quant à la loi du front d'onde, elle s'exprime par :

$$
\frac{U_{2}-U_{1}}{V_{2}-V_{1}}=2 \frac{V_{2}+V_{1}}{2} \sqrt{\frac{V_{2}^{2}+V_{1}^{2}}{2}}
$$

On peut dire qu'un observateur placé sur une section $\mathrm{du}$ canal où le régime est uniforme et traversant d'aval cn amont, l'onde de choc descendante observe cette loi.

b) Onde de choc remontant le courant. - On peut donner des lois semblables pour une onde de choc remonlant le cuurant. En particulier, la loi du front d'onde prend la forme :

$$
\frac{U_{2}}{V_{2}}=U_{1}=-2 \frac{V_{2}+V_{1}}{2} \sqrt{\frac{V_{2}^{2}+V_{1}{ }^{2}}{2}}
$$


Nous allons revenir sur cette question de l'onde remontant le courant qui est dans notre problème négative et qui ne peut se propager telle quelle.

\section{PARTICULARITÉS RELATIVES AUX ONDES DE CHOC DU CAS ÉTUDHÉ}

La suppression du barrage va laisser dévaler vers l'aval un énorme bourrelet d'eau, tandis qu'un vide va se creuser dans la retenue et s'étendra vers l'amont. Entre les deux phénomènes se propageant en sens inverse, la ligne d'eau se modifiera constamment par le jeu des ondes élémentaires venues d'amont et d'aval et qu'on étudiera d'après les lois qu'on vient d'indiquer.

Examinons comment se présentent les deux ondes de choc qui encadrent le phénomène.

\section{1. - Onde de choc positive}

Pour un observateur qui traverse l'onde de choc descendante, les lois ont été précisées.

Mais qu'enregistre un observateur accompagnant constamment l'onde de choc même?

La théorie (1) a montré que pour une onde élémentaire descendant le courant et arrivant dans une section où le fond horizontal du canal présente un décrochement $\Delta y$, la quantité $\mathrm{U}+2 \mathrm{~V}$ varie de $-\frac{\mathrm{I}}{\mathrm{U}+\mathrm{V}} \mathrm{g} \Delta y$.

En passant par continuité à un canal de pente $i$ la quantité $U+2 V$ varie de $-\frac{1}{U+V} g(-i \Delta x)$, $i$ étant égal à $-\frac{\Delta y}{\Delta x}$ avec les conventions de signes choisies.

Ce résultat reste valable si, au lieu de considérer une petite onde, on envisage une onde raide finie, car on vérife aisément que le raisonnement ne fait pas intervenir l'amplitude de l'onde mais exige simplement que le décrochement du fond $\Delta y$ soit petit.

Pour une onde de choc on pourra donc encore admettre qu'un observateur A qui lui est lié (fig. 5) noterait au cours de son déplacement :

$$
d(U+2 \mathrm{~V})=g\left(i-i \frac{\mathrm{U}^{2}}{\mathrm{~V}^{2}}\right) \frac{d x}{\mathrm{U}+\mathrm{V}}
$$

dans la mesure où, durant le parcours $d x$ l'onde ne subit aucune altération autre que celle provenant de la pente et de la perte de charge.

Or, $\Lambda$ et la tête de l'onde se déplacent à la vitesse $W$, tandis que les obscrvateurs tels que $B$ situés uu

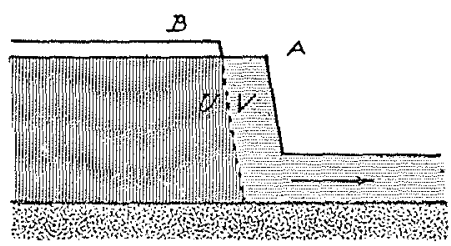

MGURE 5

peu en amont, et assez proches de $\mathrm{A}$ pour qu'ils soient caractérisés par le même $U$ et par le même $V$, possèdent la célérité $U+V$. $U+V$ est nettement supérieur à $W$ et la tête de l'onde à front raide se trouve constamment entretenue par les apports des petites ondes venant d'amont.

On néglige ces apports lorsqu'on admet qu'au cours de son déplacement, l'observateur A note la loi précédente (1).

On en tiendra compte périodiquement seulement, en utilisant des observateurs espacés qui, de temps en temps, viendront rattraper l'onde de choc. Le résultat sera qu'au moment où A sera rejoint, on gardera pour l'onde de choc le $\mathrm{U}$ et le $\mathrm{V}$ de cet observateur nouvellement arrivé en tềie de l'intumescence descendante.

L'onde de choc s'amenuisera donc tout au long de sa course et subira, par intermittence, une recrudescence destinée à la mise en compte de l'influence de l'amont.

Ce mode opératoire doit être considéré comme une simple approximation analogue à celle que l'on fait lorsqu'on résout graphiquement une équation différentielle par tronçons rectilignes successifs.

\section{2. - ONDE DE CHOC NÉCATIVE}

On sait, grâce à la théorie du ressaut, qu'une ond s négative à front raide ne peut se conserver dans un canal. Pour rester dans le cadre de la présente étude, on peut montrer comment le front raide de l'onde disparaît très vite par suite des différences de célérité des différents ćlages du mur d'eau. Pour comprendre ce mécanisme, il faut imaginer les tout premicrs instants du lâcher, vù le barrage vient de s'effacer, faisant apparaître une onde négative à l'amont, une onde positive à l'aval (fig, 6).

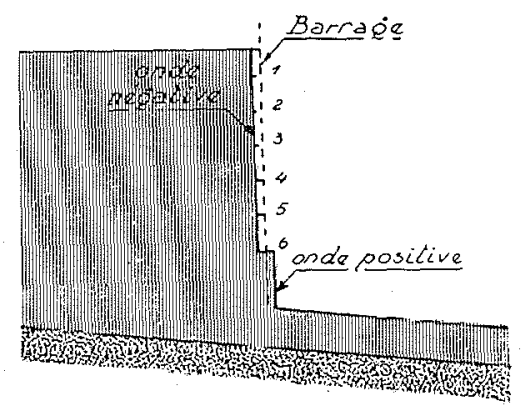

TICURE 6 
Si on considère le point le plus haut et le point le plus bas du front d'onde négative, on voit qu'étant affectés du même $U$, leur $V$ est nettement différent.

Des observateurs placés en ces points se déplaçant vers l'amont à la vitesse $|\mathrm{U}-\mathrm{V}|$ vont se trouver rapidement décalés puisque celui du haut ira beaucoup plus vite: d'où l'idée d'affecter plusieurs observateurs au contrôle de cette onde. Nous en avons disposé six, ce qui revient à fractionner l'onde négative en six ondes élémentaires qu'on étudiera séparément les unes des autres. Cette manière de voir permet d'opérer sur des ondes de petites amplitudes, avec les lois classiques.

\section{IHYSIONOMIE D'ENSEVBLE DE L'ETCUDE}

\section{MARCHE DE L'ÉPURE POUR LES PREMIERS POINTS}

Le barrage vient d'être effacé. Un mur d'eau de 9 mètres de haut va se scinder en deux pour créer les deux ondes de choc. A quelle hauteur va se faire cette scission? Telle est la première question à poser à l'épure.

Considérons les deux points $\mathrm{A}$ et $\mathrm{B}$ représentatifs des états de part et d'autre du barrage, avant le lâcher (fig. 7) : A à l'amont, $B$ à l'aval.

$B$ va voir arriver une onde positive (UV) qui obćit à la loi des ondes de choc descendantes :

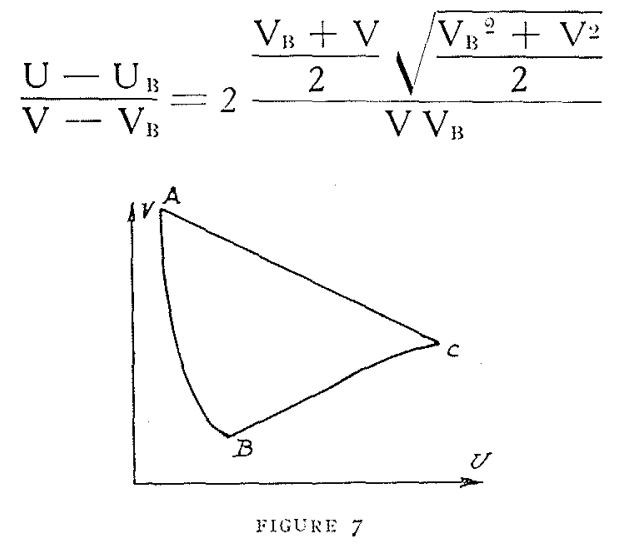

$A$ va voir arriver une onde négative de même $U V$ qui obéit à la loi des ondes de choc remontant le courant :

$$
\frac{U-U_{A}}{V-V_{A}}=2 \frac{\frac{V_{A}+V}{2} \sqrt{\frac{V_{A}^{2}+V^{2}}{2}}}{V V_{A}}
$$

Praliquement, ces deux caractéristiqucs diffèrent très peu des droites de pente +1 qu'on aurait pris si on s'était trouvé en présenre d'nndes dlćmentaires. Elles se coupent en $C$ qui représente l'état au barrage, imm:diatement après le lâcher.

\section{LECTURE DES ÉPURES}

Ces précisions étant données, nous invitons le lecteur à jeter un coup d'œil sur les différentes épures. Pour en rendre la lecture moins ardue, nous le convions à se représenter la petite course de relais suivante, d'un genre un peu particulier.

On dispose des observateurs sur une dizaine de kilomètres de chaque côté du barrage. Très près les uns des autres au voisinage du barrage, leur écartement augmente à mesure qu'on s'en éloigne.

Dans le bief aval, les observateurs se dénomment 1 , $2,3,4$, etc.; vers l'amont, $a, b, c$, d, etc. (fig. 8).

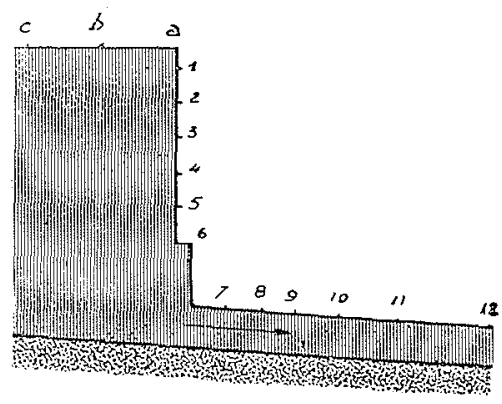

FIOURE 8

Nous examinerons à part : $a, 1,2,3,4,5$ et 6 qui jouent un rôle un peu particulier. Pour les autres, la consigne est la suivante : les observateurs se mettent en mouvement lorsque le barrage étant effacé la perturbation ainsi produite vient les toucher. Ils chevauchent alors non pas cette onde qui est venue leur donner le signal, mais celle qui, caractérisée par les mêmes $U$ et $\mathrm{V}$, repart aussitôt en sens inverse, et ils ne la quittent plus.

Les observateurs chiffres placés à l'aval se meuvent vers l'amont à la vitesse $|\mathrm{U}-\mathrm{V}|$.

Les observateurs lettres placés à l'amont se déplacent vers l'aval à la vitesse $\mathrm{U}+\mathrm{V}$.

Voyons, maintenant, le rôle des observateurs spéciaux.

a situś au dśpart, au barrage même, se déplace vers l'aval avec le front de l'onde raide dont nous avons déjà donnś la célérité :

$$
W=U_{1}+\frac{V_{0}}{V_{1}} \sqrt{\frac{V_{1}^{2}+V_{2}^{2}}{2}}
$$

On verra qu'il sera rattrapé par $b$. A ce moment, $a$ cst mis hors de course et lègue à $b$ sa célérité particulière; à son tour $b$ se fera rejoindrc par $c$ et lui cidera sa place, et ainsi de suite.

Quant à $1,2,3,4,5$ et 6 , ce sont les six observateurs qui choisissent comme position de départ des altitudes différentes sur l'onde de choc nćgative. 1 est placé en haut de ce front d'onde, 6 tout en bas. Comme leur 
PLANCHE 1

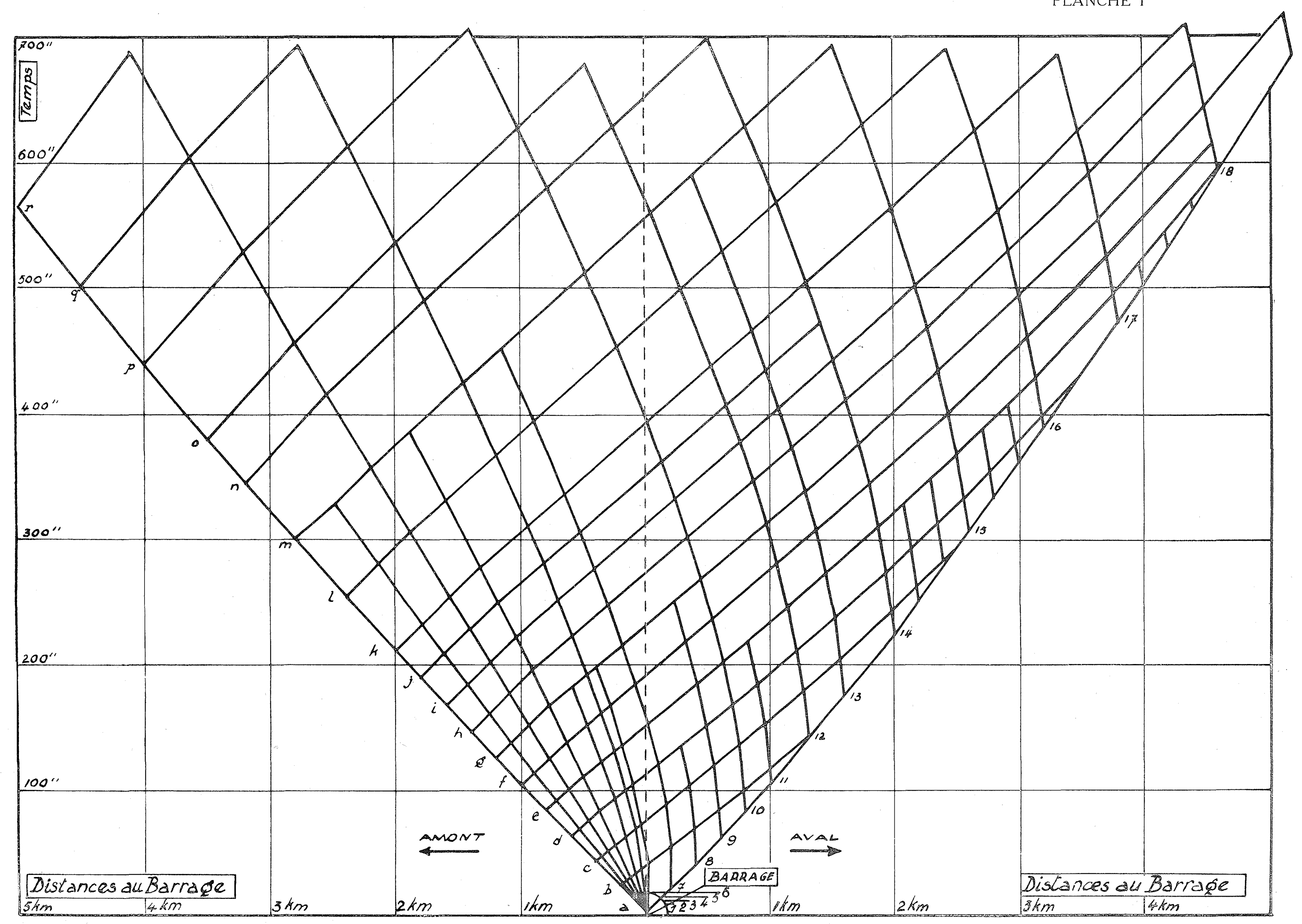




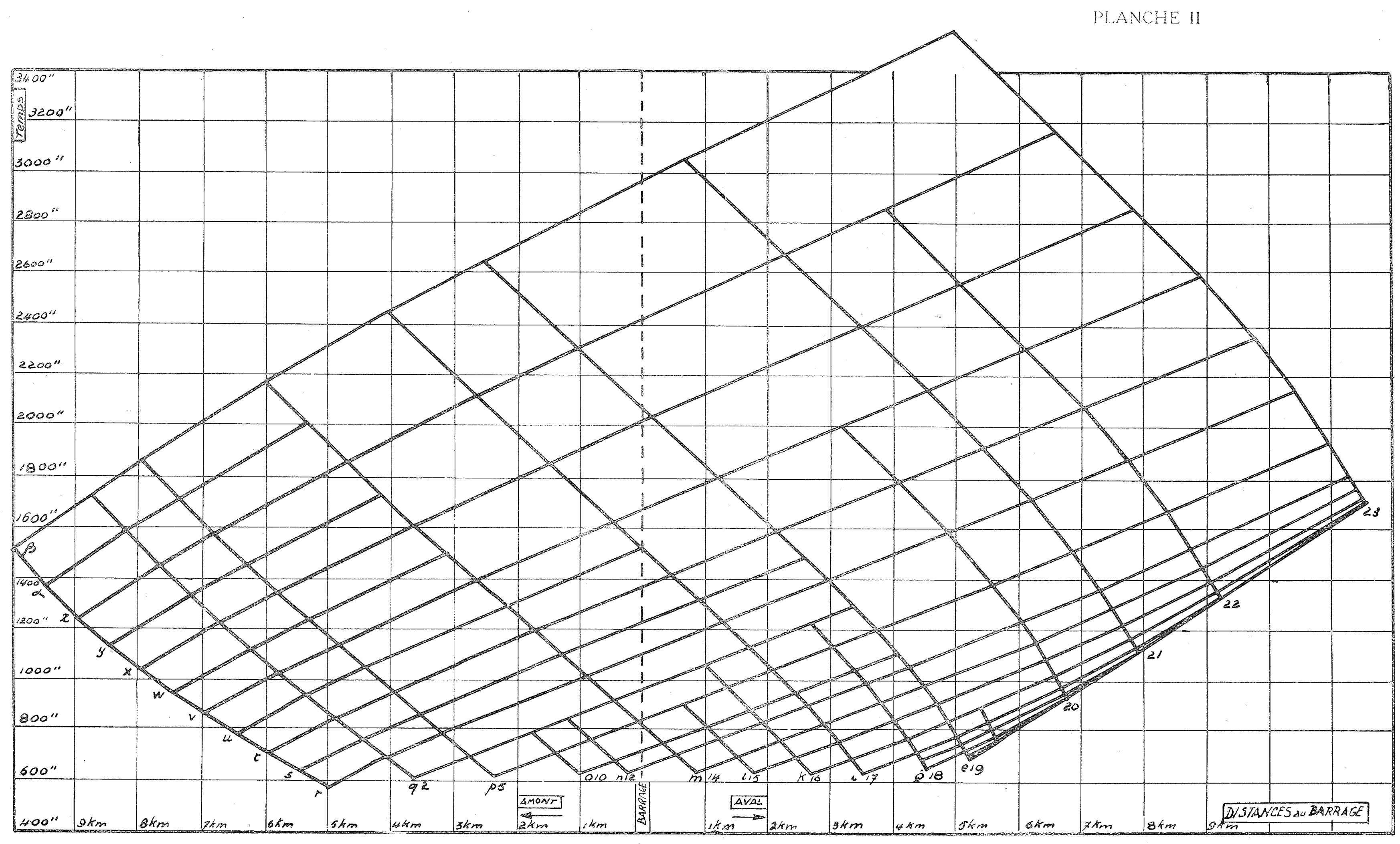




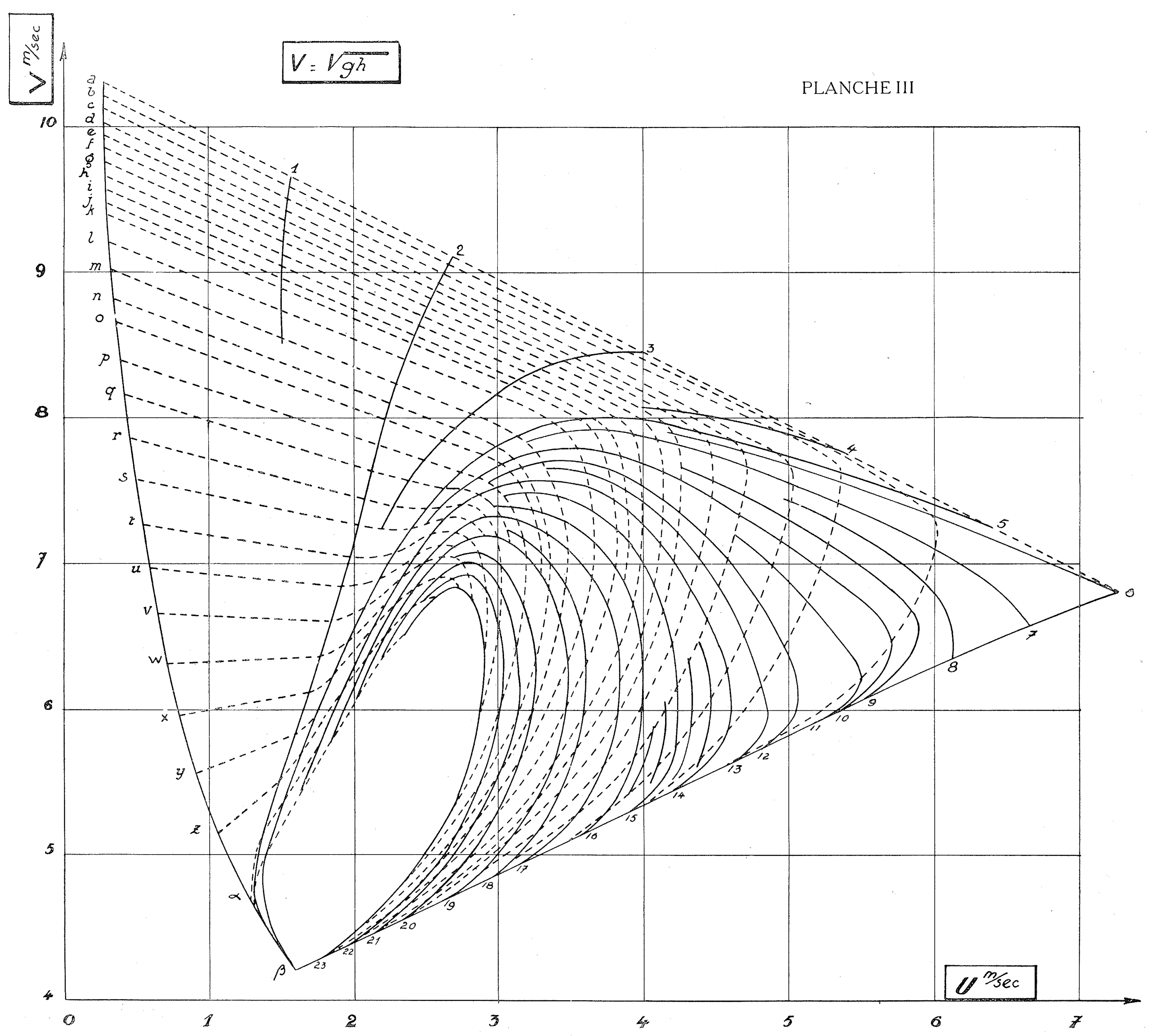


PLANCHE IV

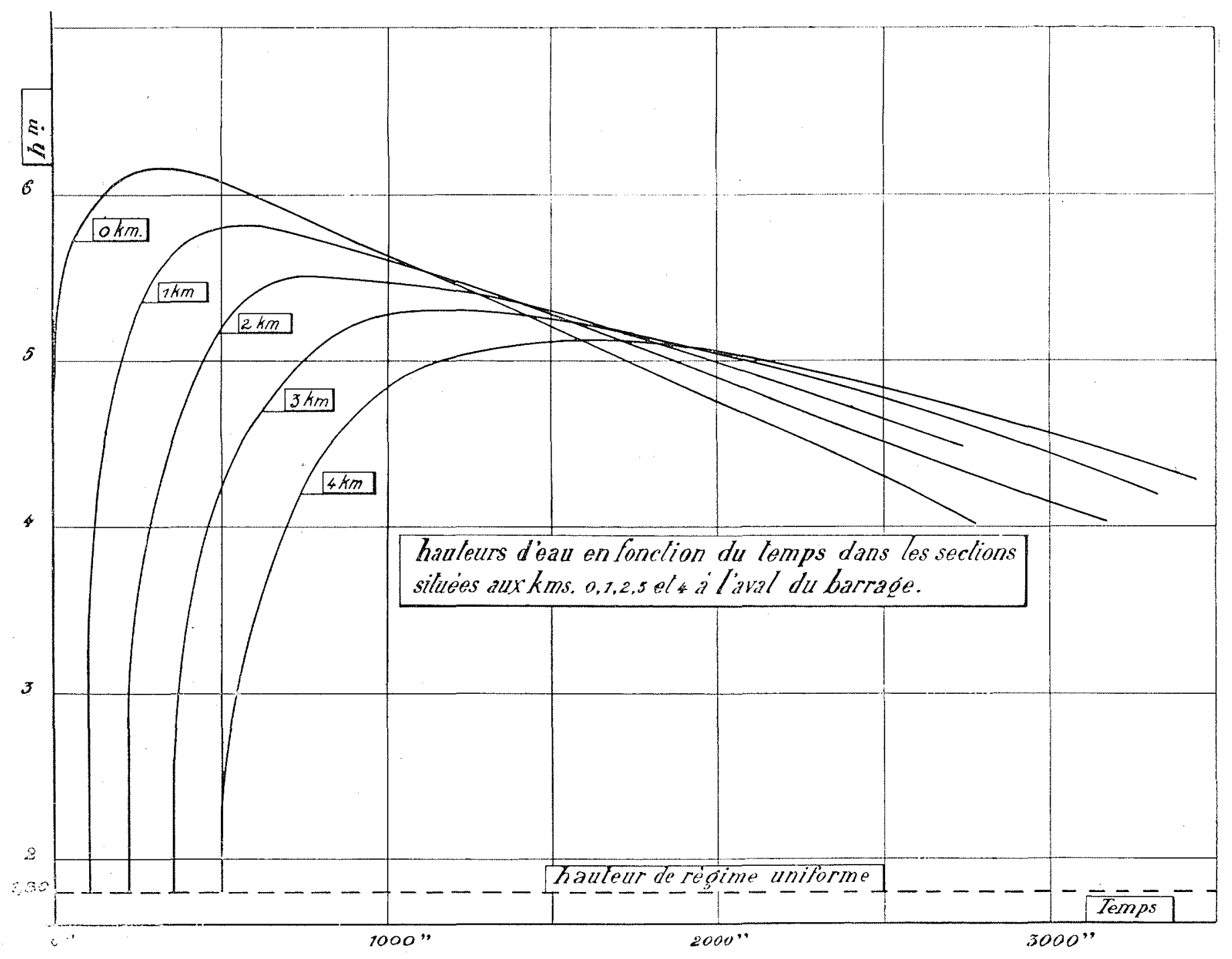

PLANCHE V

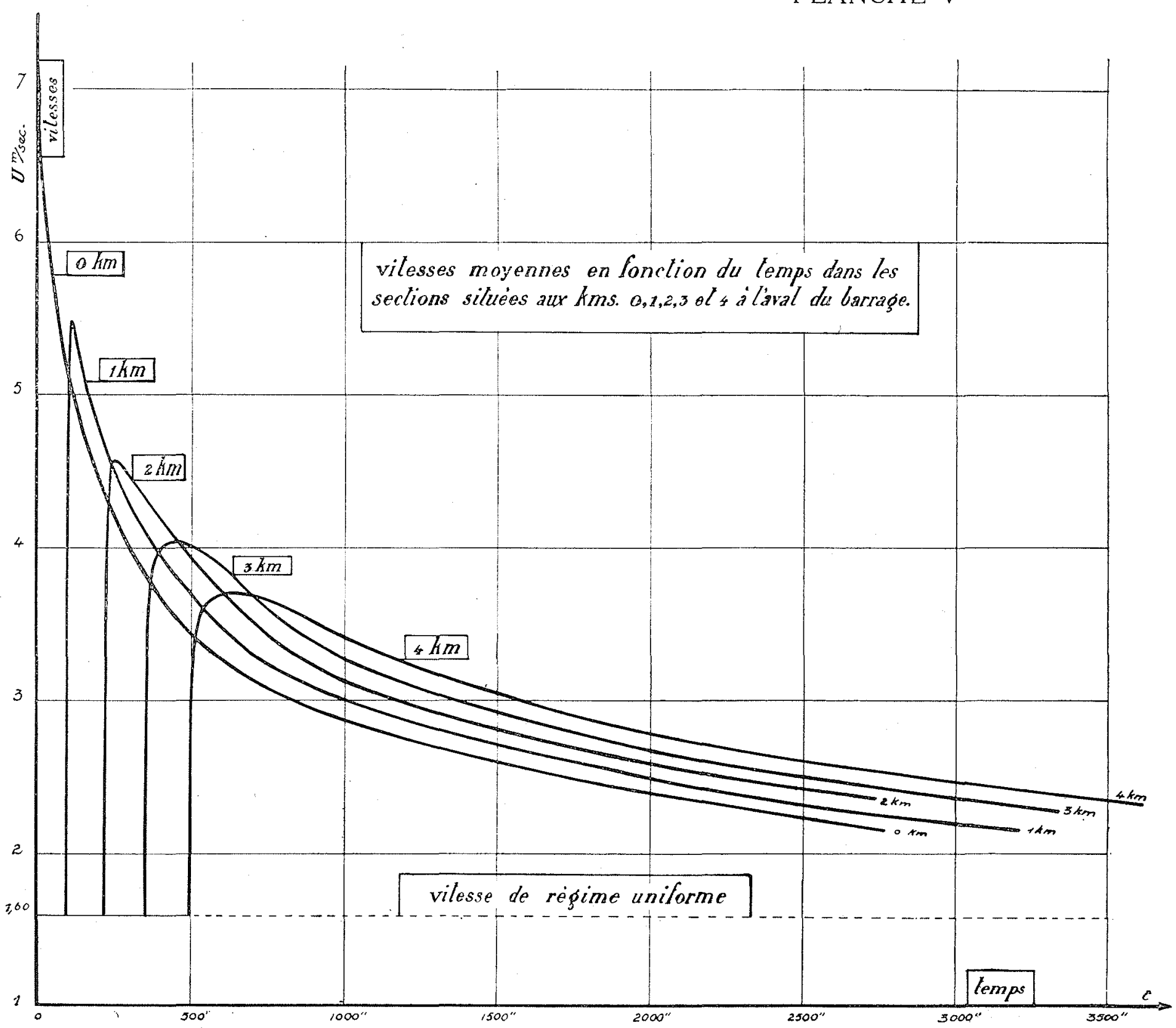


PLANCHE VI

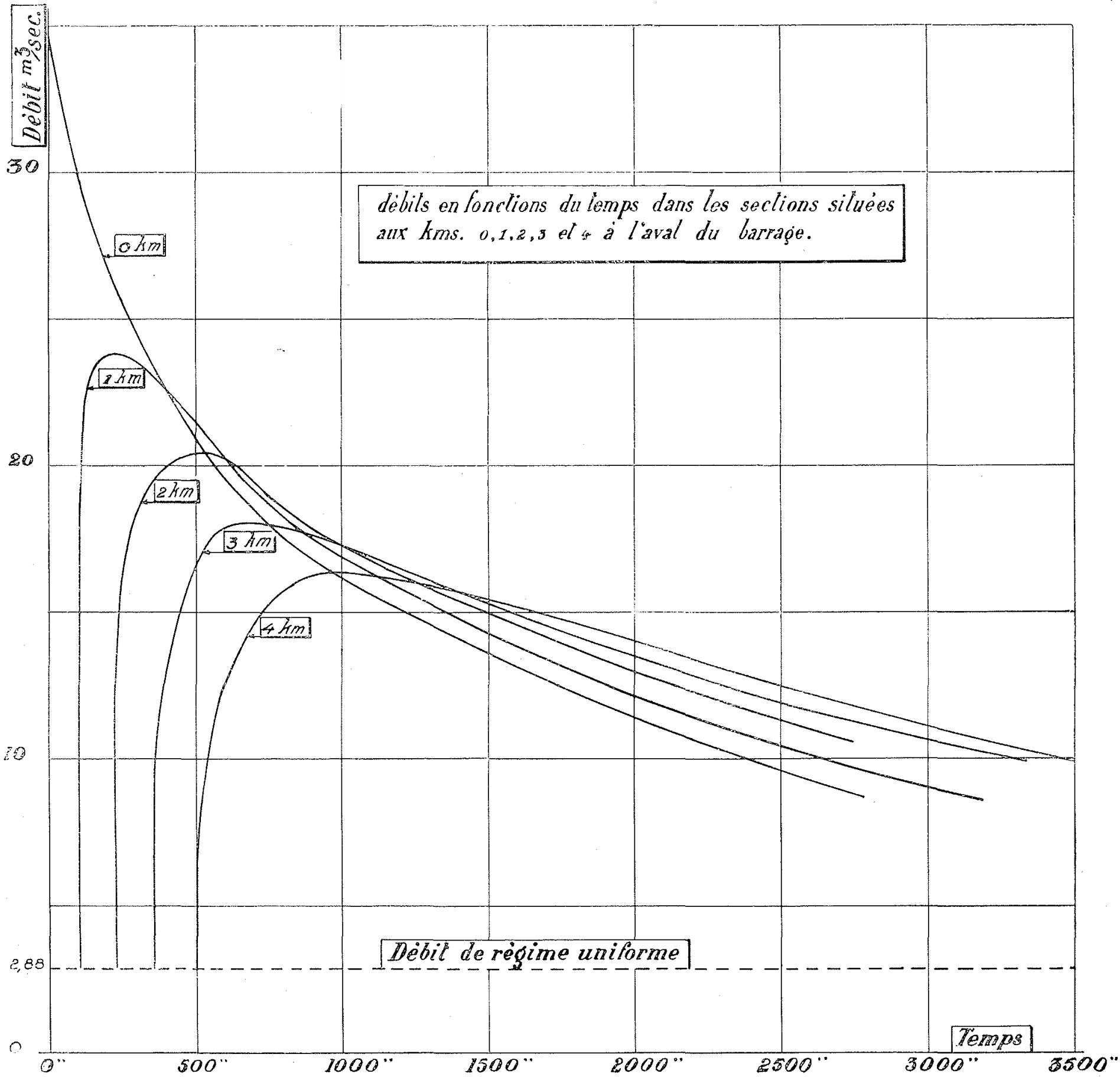



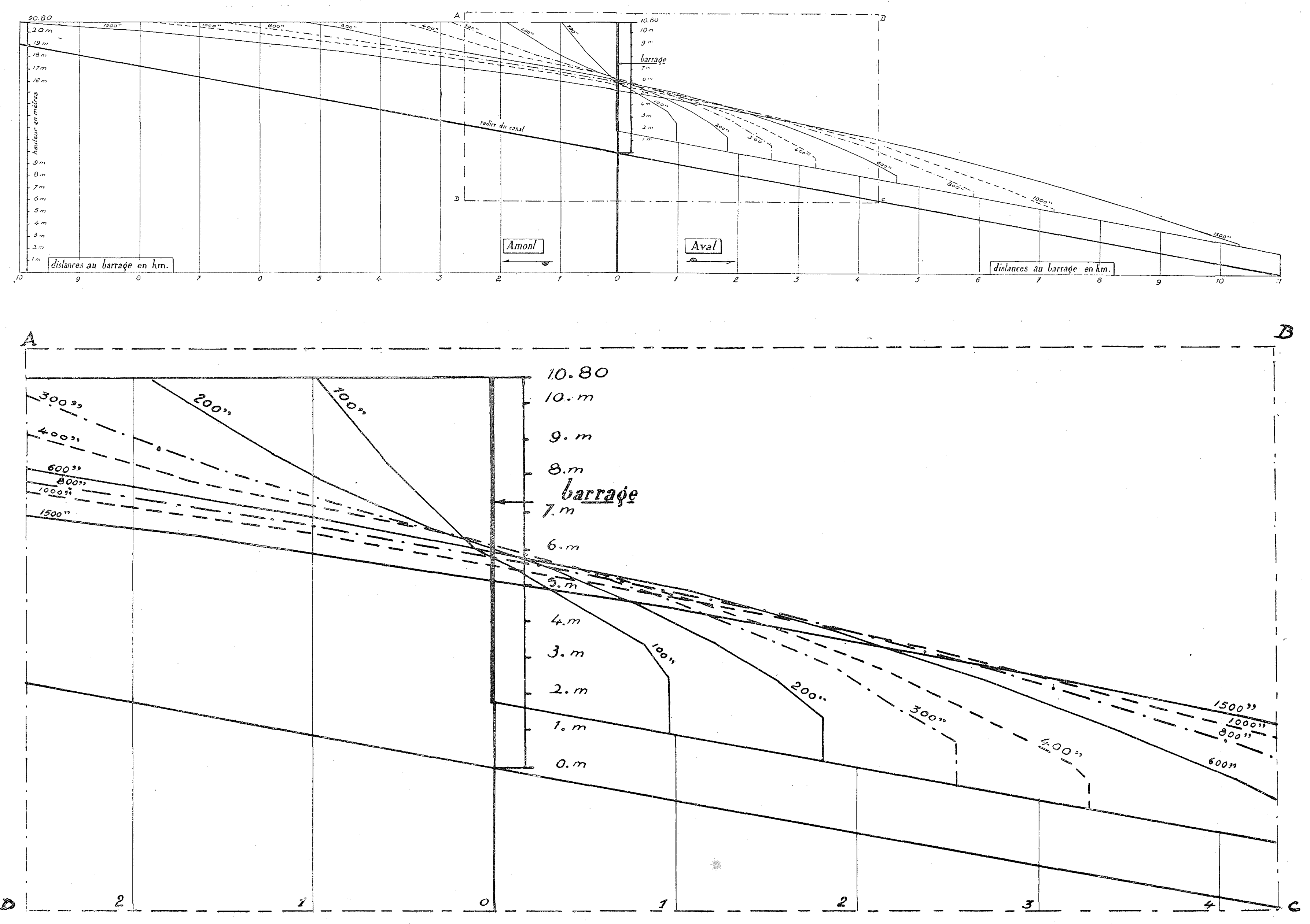
cćlórité est $|\mathrm{U}-\mathrm{V}| \quad 1$ remontera plus vite que 2 , ce dernier plus vite que 3 et ainsi de suite jusqu'à 6 qui sera le plus lent du groupe.

Les observateurs lettres et les observateurs chiffres vont donc se croiser. Dans un plan de coordonnées $x, t$, nous suivons leurs déplacements dans le temps (planches I et II).

Pour un observateur donné, l'ensemble des points représentatifs des états ( $\mathrm{UV}$ ) rencontrés, forme une courbe continue qui n'est autre que la loi caractéristique de l'observateur considéré. On voit donc que la caractéristique simple $\mathrm{U}= \pm 2 \mathrm{~V}$ est devenue, du fait de la pente du canal et de la perte de charge, une loi $\mathrm{U}=f(\mathrm{~V})$ plus complexe. C'est le carnet de bord et à chaque rencontre, on fera le point: telle vitesse, tel $V$, donc telle hauteur (planche III).

Deux observateurs de classe différente ne se rencontrent qu'une fois.

Le plan $x t$ donne le lieu et l'époque de la rencontre; le plan U V indique alors la vitesse et la hauteur qu'ils ont enregistrées.

Dans le plan $U V$, on remarque que des observateurs successifs se propageant dans la même direction, ont tendance à enregistrer des états identiques. Autrement dit leurs lois caractéristiques cherchent à converger. Cela signifie que, sur une grande partie du canal, l'état reste sensiblement le même et qu'on dispose de plusieurs observateurs pour enregistrer les mêmes résultats. Le grand ordonnateur du spectacle suspend alors l'un d'eux, lorsqu'il estime que la précision de l'épure n'en est pas affectée; ceci explique l'arrêt brusque de certaines courbes caractéristiques.

\section{CONSEILS GRAPHIQUES POUR LA CONDUITE DES ÉPURES}

\section{PRECAUTIONS POUR OBTENIR UNE BONNE PRECISION}

Considérons deux observateurs $\mathrm{A}$ et $\mathrm{B}$ allant à l'encontre l'un de l'autre, et soient $A$ et $B$ leurs points représentatifs dans le plan $U \mathrm{~V}$ et $a, b$ dans le plan $x t$ (fig. 9).

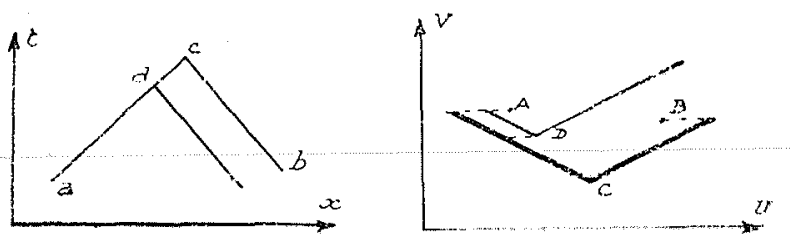

FIGURE 9
Pour $\mathrm{A}$ on a décalé sa caractéristique parallèlement à $\mathrm{OU}$ de la quantité :

$$
g\left(i-i \frac{U_{A}^{2}}{V_{A}{ }^{2}}\right)\left(t_{C}-t_{A}\right)
$$

Pour B de

$$
\mathrm{g}\left(i-i \frac{\mathrm{U}_{\mathrm{B}}^{2}}{\mathrm{~V}_{\mathrm{B}}^{2}}\right)\left(t_{\mathrm{C}}-t_{\mathrm{B}}\right)
$$

On conçoit qu'il faille prendre des intervalles de temps assez petits pour que de $A$ à $C$ ou de $B$ à $C$ le $U$ et le $V$ ne changent pas trop.

Graphiquement, on aura le droit d'aller de $A$ en $C$ si on est certain qu'en prenant un intermédiaire $D$, l'état obtenu à partir de $B$ et $D$ est bien celui de $C$.

\section{ESPACEMENT DES OBSERVATEURS}

Dans la mesure où on peut considérer $\lambda$ comme constant, on voit que le décalage par unité de temps est une fonction simple de $\mathrm{U} / \mathrm{V}$ (linéaire en $\mathrm{U}^{2} / \mathrm{V}^{2}$ ). On pourra tracer, au préalable, une courbe de décalage unitaire $\varphi$ en fonction de U/V (fig. 10).

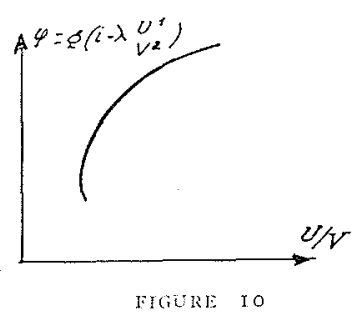

Si, dans le plan U, V, la loi A B C suivie par un observateur se rapproche d'une droite du faisceau issu de l'origine (fig. 11), c'est que, pour cet observateur, $\mathrm{U} / \mathrm{V}$ reste sensiblement constant; le décalage unitairc à appliquer à son point représentatif sera aussi à peu près constant et on pourra lui faire parcourir de longs trajets en une seule fois.

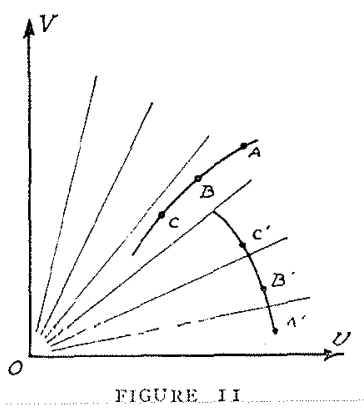

Si, au contrairc, la loi $\mathrm{A}^{\prime} \mathrm{B}^{\prime} \mathrm{C}^{\prime} \mathrm{d}^{\prime}$ un observateur rencontrc le faisceau des droites issues de l'origine plutôt 
orthogonalement, il faudra user de prudence et limiter les parcours successifs de l'observateur.

Une autre remarque permet d'accélérer la marche de l'épure.

D'un observateur $A$ et d'un observateur $B$ allant à l'encontre l'un de l'autre, nous connaissons déjà, lorsque l'épure est un peu avancée, les enregistrements successifs dans le plan UV (fig. 12); ce sont respectivement $A_{1} A_{2} A_{3} A_{4}$ et $B_{1} B_{2} B_{3} B_{4}$.

On peut, en extrapolant ces lois, déterminer d'avance approximativement l'état qu'ils trouveront en $A_{\bar{j}}^{\prime}$ où ils se rencontreront.

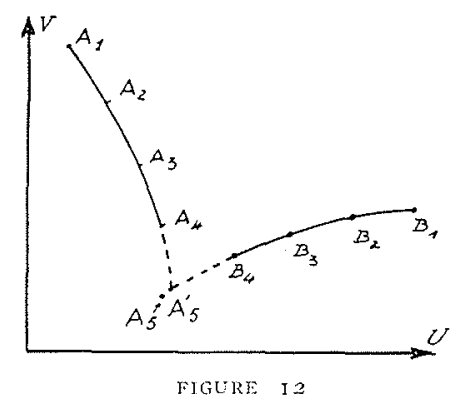

Pour le décalage unitaire à faire subir à la caractéristique issue de $\mathrm{A}_{4}$ on prendra la moyenne des décalages unitaires applicables en $A_{4}$ et $A_{5}^{\prime}$ soit :

$$
\frac{\theta_{A}+C_{A^{\prime}}^{\prime}}{2}\left(t_{A_{5}}^{\prime}--t_{A_{1}}\right)
$$

Pour la caractéristique issue de $B$ le décalage sera :

$$
\frac{A_{i}^{\prime}+C_{B 4}}{2}\left(t_{A 5}^{\prime}-t_{134}\right)
$$

Ces deux caractéristiques se couperont au voisinage de $A_{5}^{\prime}$, en un point $A_{5}$ qui est la véritable intersection des deux caractéristiques considérées.

On attachera à cette remarque toute l'importance qu'elle mórite, car elle permet d'espacer les observateurs ct réduit au moins de moitié le nombre de points à d:terminer graphiquement.

\section{EXPLOITATION ET COMMENTAIRE des résultats}

En coupant le plan $x t$ par les droites $x=$ Cte on d'termine par interpolation une série de points dont on trouve les correspondants clans le plan UV . On peut, par suite. tracer les courbes de hauteur, de vilesse et de dśbit, en fonction du temps, pour une section donnée (planches IV, $V$ et VI).

On remarquera que, pour chague section, les maxima de hauteur, de vitesse et de débit ont lieu dans l'ordre suivant : d'abord maximum de vitesse, puis maximum do débit, puis, beaucoup plus tard, maximum de hauteur.

En coupant le plan $x t$ par les droites $t=$ Cte on peut tracer également les lignes d'eau à des instants donnés (planche VII). Elles donnent une idée de l'évolution générale des intumescences.

La question se pose, maintenant, de la précision avec laquelle l'épure fournit ces résultats.

Le test suivant, facile à effectuer, permet de s'en rendre compte.

$\mathrm{Si}$ on considère deux sections du canal, on peut déterminer par deux moyens différents la quantité d'eau qui s'y est accumulée entre deux temps donnés.

Sur le graphique des lignes d'eau, l'aire comprise entre les deux sections et les deux lignes d'eau aux deux temps donnés représente le volume considéré (fig. 13).

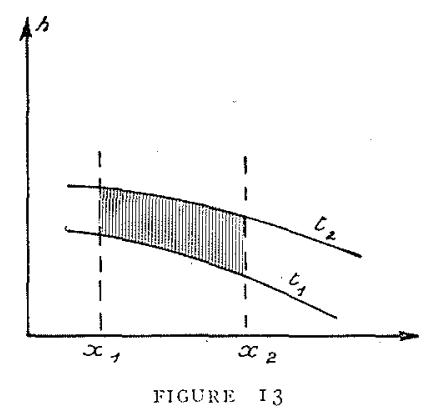

Sur la courbe débit-temps, l'aire comprise entre les deux verticales correspondant aux deux temps donnés et les deux courbes de débit des deux sections choisies représente le même volume d'eau (fig. 14).

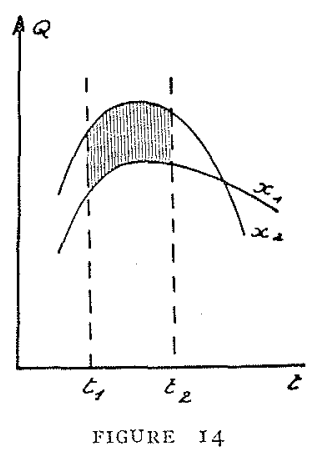

On sait que la méthode graphique traduit de manière équivalente les ćquations do Saint-Venant; celles-ri comprennent l'ćquation dynamique et l'équation de continuité, conditions qui sc trouvent toutes deux imbriquées dans les règles des caractéristiques.

Les ćpurcs constituent par conséquent la solution approchée des équations de Saint-Venant répondant aux 
conditions initiales et aux limites imposées, et le test dont nous venons de parler revient, en somme, à jauger l'approximation avec laquelle cette solution vérifie l'équa. tion de base.

Le lecteur pourra lui-même, à l'aide d'un planimètre, faire les vérifications qu'il jugera nécessaires.

Pour les sections voisines du barrage, il y a quelque divergence, mais si on choisit des sections assez écartées et des intervalles de temps assez longs, on trouve un bonne concordance des résultats. Ainsi, le volume accumulé entre les kilomètres 1 et 4 pendant les 1.500 premières secondes de lâcher atteint, avec les deux procédés, respectivement 10.390 et $10.130 \mathrm{~m}$, soit un écart de $2,5 \%$. Si le volume réel est compris entre ces deux valeurs, en adoptant pour valeur approchée de ce volume la moyenne de ces deux valeurs, on commet une erreur inférieure à $1,25 \%$.

\section{CONCLUSIONS}

Nous espérons que l'exemple d'application du calcul graphique des intumescences que nous venons d'exposer aura permis de se faire une idée correcte des possibilités de la méthode, de ses difficultés et du résultat qu'elle est susceptible de donner.

Certaines de ces difficultés proviennent du problème que nous avons choisi de traiter; l'effacement brutal d'un barrage produit une onde qui déborde un peu et, particulièrement au dśbut, le cadre des écoulements graduellement variés; on sait que l'équation de Saint-Venant, dont nos épures constituent une intégration graphique, supposent les courbures de la surface libre négligeables; il y a lieu de souligner que cette condition n'est pas, dans le cas présent, complètement remplie et que le calcul constitue, de ce fait, une schématisation de la réalité.

C'est notamment le cas pour l'étude de l'étalement de l'onde négative par l'artifice de la d'́composition en petites ondes élémentaires; de même en ce qui concerne l'onde positive, on sait qu'elle s'accompagne d'ondulations dont l'approximation de l'écoulement graduellement varió ne rend pas compte: l'expérience montre toutefois que la ligne d'eau obtenue par le schéma du calcul coincide sensiblement avec une ligne moyenne tracée au travers des ondulations.

En ce qui concerne la mise en oeuvre des épures, i] ne faut pas se dissimuler qu'elle représente un travail assez long et beaucoup plus délicat que pour le coup de bélier. Malgré la clarté apportée par la représentation graphique, des erreurs dans les décalages peuvent passer inaperçues, tout au moins au commencement de l'épure où l'on ne voit pas encore la forme des courbes UV suivies par les observateurs.

Cette complication est d'ailleurs dans la nature des choses et aucune méthode de calcul ne saurait l'éliminer complètement; en contre-partie du travail effectué, l'épure donne avec une bonne approximation une description complète et dátaillée du phénomène aux différents lieux et aux différents instants.

Comme nous l'avons dit, le problème que nous venons d'examiner s'est trouvé posé par les opérations militaires qui ont abouti à la Victoire : il s'agissait de prévoir les periurbations que l'ennemi était susceptible d'apporter à l'écoulement du Rhin, par la manæuvre des barrages à la frontière germano-suisse; l'étude théorique qui précède se situe à l'origine de cette recherche de grande ampleur; elle permit de dégrossir le problème, d'orienter et de contrôler la recherche expérimentale à laquelle il appartint, en définitive, d'apporter la réponse complète aux difficiles et complexes questions posées. 


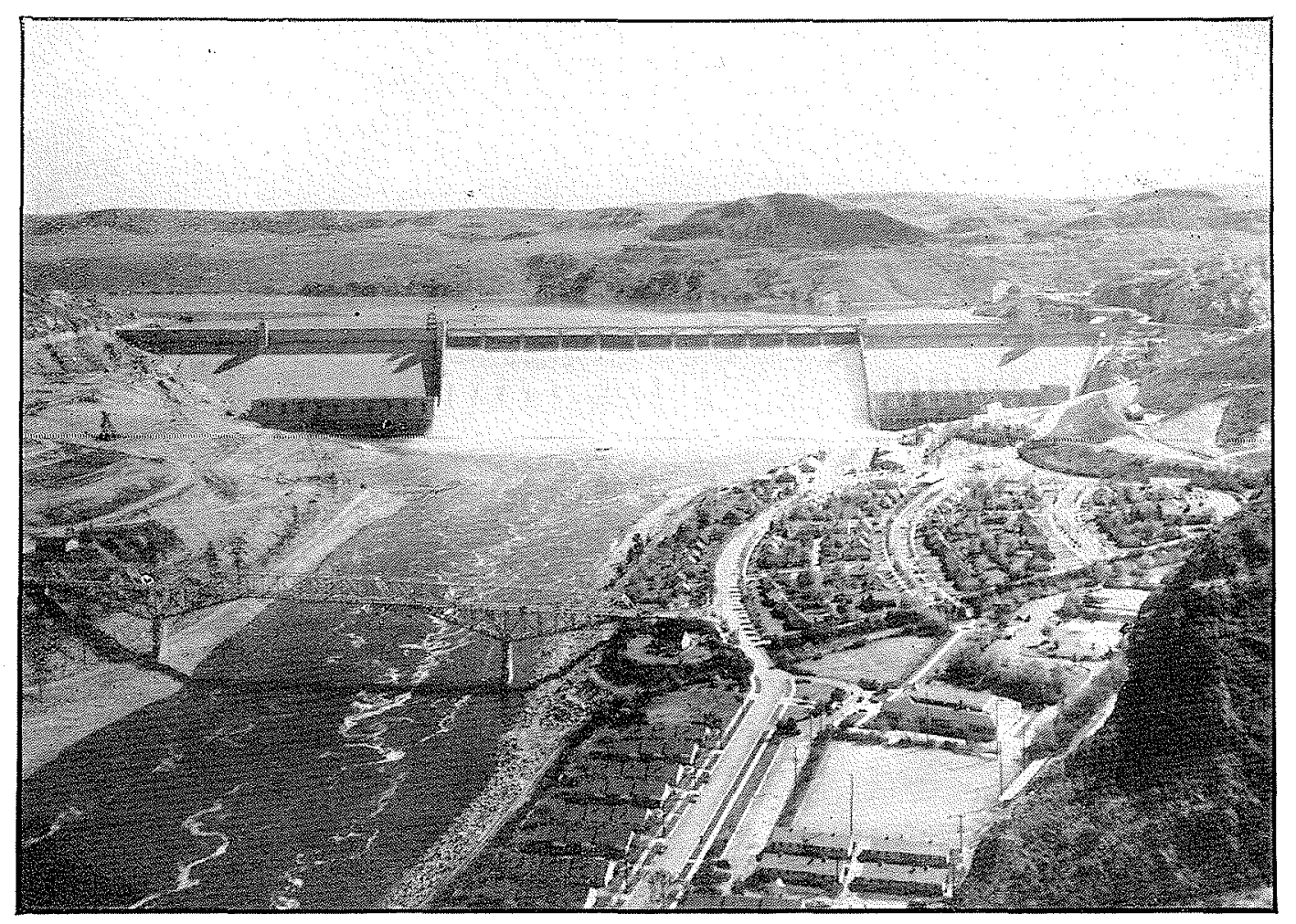

LE BARRAGE DE GRAND COULEE AUX ÉTATS-UNIS

VUE DE JOUR ET VUE DE NUIT

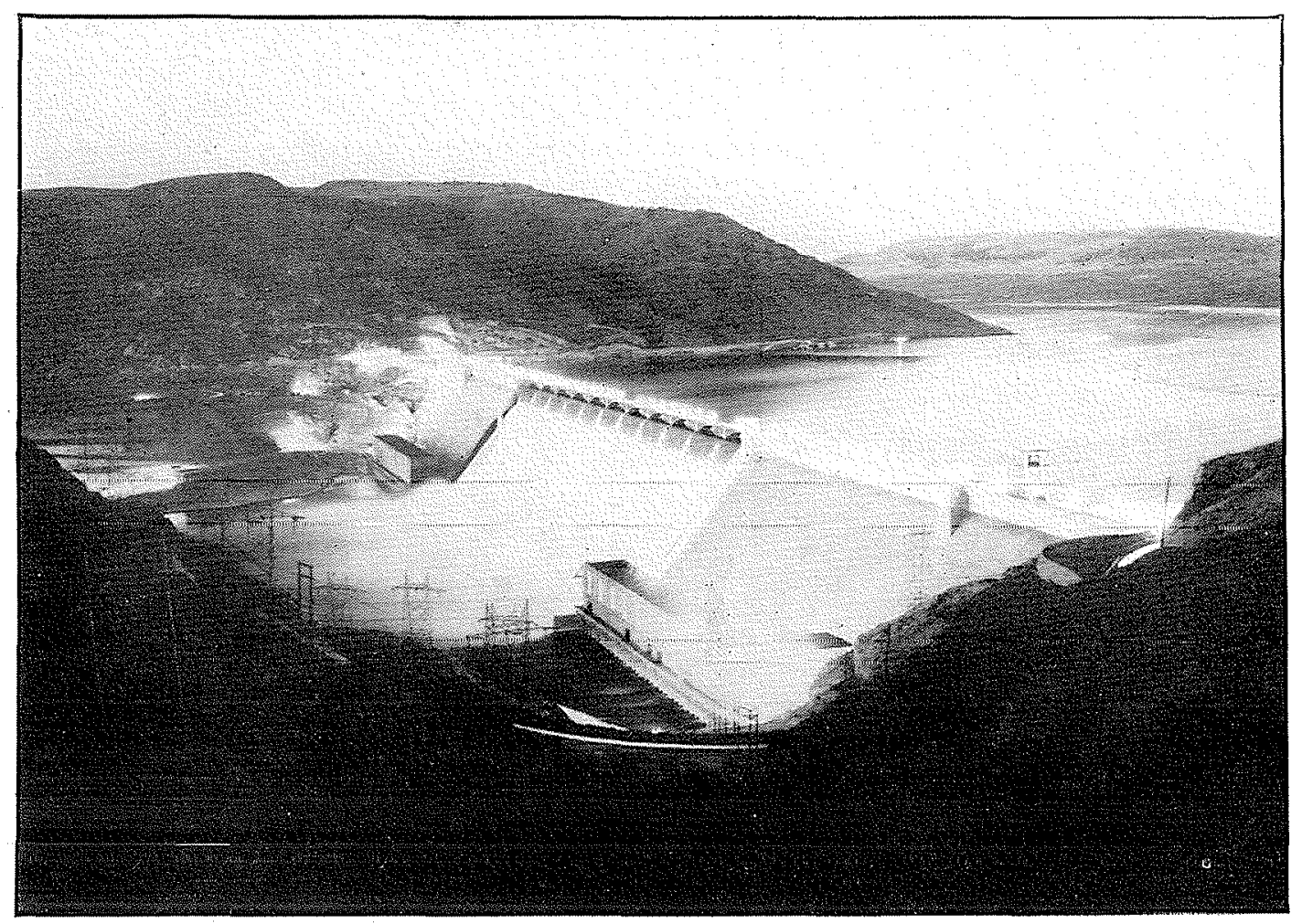

PHOTU U. S. 1. : :s. 